\title{
Safety and Tolerability of Bintrafusp Alfa, a Bifunctional Fusion Protein Targeting TGF $\beta$ and PD-L1, in Asian Patients with Pretreated Recurrent or Refractory Gastric Cancer
}

Yoon-Koo Kang ${ }^{1}$, Yung-Jue Bang ${ }^{2}$, Shunsuke Kondo ${ }^{3,4}$, Hyun Cheol Chung ${ }^{5}$, Kei Muro $^{6}$, Isabelle Dussault ${ }^{7}$, Christoph Helwig ${ }^{8}$, Motonobu Osada ${ }^{9}$, and Toshihiko Doi ${ }^{10}$

\section{ABSTRACT}

Purpose: Patients with advanced gastric/gastroesophageal junction cancer (GC/GEJC) have limited treatment options after firstline therapy. Bintrafusp alfa is a first-in-class bifunctional fusion protein composed of the extracellular domain of the TGFßRII receptor (a TGF $\beta$ "trap") fused to a human IgG1 antibody against programmed death ligand 1 (PD-L1), potentially offering a new treatment approach for these patients. We report results for bintrafusp alfa in GC/GEJC.

Patients and Methods: Asian patients with recurrent GC/GEJC for whom standard therapy does not exist or for whom standard therapy has failed enrolled in this expansion cohort of an ongoing phase I trial and received bintrafusp alfa 1,200 mg once every 2 weeks until disease progression, unacceptable toxicity, or withdrawal. The primary objective was to assess safety/tolerability.

\section{Introduction}

Gastric cancer, including gastroesophageal junction cancer (GEJC), is the fifth most common cancer in the world and the third leading cause of cancer-related death worldwide (1). Approximately $60 \%$ of gastric cancer cases occur in eastern Asia, which has the highest estimated mortality rate for gastric cancer worldwide (1). The prognosis is often poor because gastric cancer is an aggressive disease and, except in Japan and South Korea, is mostly diagnosed in advanced

${ }^{1}$ Department of Oncology, Asan Medical Center, University of Ulsan College of Medicine, Seoul, South Korea. ${ }^{2}$ Department of Internal Medicine, Seoul National University College of Medicine, Seoul, South Korea. ${ }^{3}$ Department of Experimental Therapeutics, National Cancer Center Hospital, Tokyo, Japan. ${ }^{4}$ Department of Hepatobiliary and Pancreatic Oncology, National Cancer Center Hospital, Tokyo, Japan. ${ }^{5}$ Medical Oncology, Yonsei Cancer Center, Yonsei University College of Medicine, Seoul, South Korea. ${ }^{6}$ Aichi Cancer Center Hospital, Nagoya, Japan. ${ }^{7}$ EMD Serono Research \& Development Institute, Inc., Billerica, Massachusetts; a business of Merck KGaA, Darmstadt, Germany. ${ }^{8}$ Merck KGaA, Darmstadt, Germany. ${ }^{9}$ Merck Biopharma, Tokyo, Japan; an affiliate of Merck KGaA, Darmstadt, Germany. ${ }^{10}$ National Cancer Center Hospital East, Chiba, Japan.

Note: Supplementary data for this article are available at Clinical Cancer Research Online (http://clincancerres.aacrjournals.org/).

Y.-K. Kang and Y.-J. Bang contributed equally as the co-first authors of this article.

Corresponding Author: Yung-Jue Bang, Seoul National University College of Medicine, 101 Daehak-ro, Jongno-gu, Seoul 03080, South Korea. Phone: 82-26925-6203; Fax: 82-2-6925-6204; E-mail: bangyj@snu.ac.kr

Clin Cancer Res 2020;26:3202-10

doi: 10.1158/1078-0432.CCR-19-3806

(C)2020 American Association for Cancer Research.
Results: By July 23, 2018, 31 heavily pretreated patients received bintrafusp alfa for a median of 10.1 weeks; 3 patients remained on treatment. Six patients (19\%) experienced grade 3 treatment-related adverse events (AE); no grade 4 events occurred. One on-treatment death occurred (sudden death); rupture of a preexisting thoracic aortic aneurysm was the suspected cause. Ten patients (32\%) had immunerelated AEs. The confirmed objective response rate per independent review committee was $16 \%$; disease control rate was $26 \%$. Median duration of response was 8.7 months (range, 2.4-12.4+). Responses occurred irrespective of PD-L1 expression or microsatellite instability status and appeared to correlate with high tumor TGFB1 levels.

Conclusions: In this first evaluation in Asian patients with heavily pretreated advanced GC/GEJC, bintrafusp alfa demonstrated a manageable safety profile and clinical activity.

stages $(2,3)$. Many patients present with metastatic disease at diagnosis, with progression-free survival (PFS) rarely exceeding 6 months with most first-line chemotherapy treatments (3-5).

Tumor characteristics known to have prognostic or predictive importance in GC/GEJC include PD-L1 expression, microsatellite instability (MSI) status, mutational load, Epstein-Barr virus (EBV) infection, and immune phenotype (2,5-9). In addition, many recent anticancer treatments focus on modulating the tumor microenvironment to prevent cancer progression (10). TGF $\beta$, a cytokine that can act in the tumor microenvironment, can promote epithelialmesenchymal transition, increase angiogenesis, and mediate tumor cell transition to a more stem-like and invasive cell phenotype $(11,12)$. TGF $\beta$-mediated signaling can suppress immune surveillance and is associated with larger tumor volume, disease progression, and reduced survival for patients with gastric cancer (11-16).

Currently, there is no standard third-line therapy for GC/GEJC. International treatment recommendations include chemotherapy regimens, such as trifluridine/tipiracil (TAS-102) and PD-1 inhibitors, as well as second-line treatment options that were not used in prior therapy $(2,5)$. Data from large phase II and III trials show median overall survival (OS) ranging from 5.3 to 6.7 months and objective response rates (ORR) of $4 \%$ to $12 \%$ with these agents in unselected patients with pretreated advanced GC/GEJC (17-20). In addition, recent phase III studies of immune checkpoint inhibitors (ICI) compared with chemotherapy failed to meet their primary endpoint of OS, highlighting the need to investigate new treatment strategies in GC/GEJC $(20,21)$.

TGF $\beta$ is a potent immune suppressor and has been implicated in decreasing responsiveness to ICIs (22). In fact, the combined use of a TGF $\beta$ blocker and an ICI has shown an elevated effect over either agent alone (22-26). Because of this, inhibiting TGF $\beta$ activity in the tumor 


\section{Translational Relevance}

Gastric and gastroesophageal junction cancers (GE/GEJC) represent a common and lethal group of cancers. Although immune checkpoint inhibitors can offer therapeutic benefit, simultaneously targeting multiple cancer-related pathways, such as TGF $\beta$ and PD-L1, with a single agent may enhance the antitumor activity observed when targeting a single pathway individually. We report results from the first phase I evaluation of a bifunctional agent targeting both TGF $\beta$ and PD-L1 in GC/GEJC. Bintrafusp alfa is a first-in-class bifunctional fusion protein composed of the extracellular domain of the TGF $\beta$ RII receptor (a TGF $\beta$ "trap") fused to a human IgG1 antibody blocking PD-L1. In this ongoing trial, bintrafusp alfa had a manageable safety profile in Asian patients with heavily pretreated advanced GC/GEJC that was consistent with previous reports for this treatment in solid tumors. In addition, an objective response rate of $16 \%$ and prolonged duration of response [median, 8.7 months (range, 2.4-12.4+)] were observed with bintrafusp alfa.

microenvironment while simultaneously blocking an additional immunosuppressive cellular mechanism, such as the PD-L1 pathway, may provide a novel treatment approach and potentially enhance clinical benefit compared with established anti-PD-(L)1 monotherapies $(27,28)$.

Bintrafusp alfa (M7824) is a first-in-class bifunctional fusion protein composed of the extracellular domain of the human TGF $\beta$ receptor II (TGF $\beta$ RII or TGF $\beta$ “trap") fused via a flexible linker to the C-terminus of each heavy chain of an IgG1 antibody blocking PD-L1 (27). Preclinical studies in murine models indicate that bintrafusp alfa can accumulate in the tumor microenvironment and reduce intratumoral TGF $\beta$ signaling and improve antitumor activity compared with either a TGF $\beta$ "trap" or an anti-PD-L1 antibody alone $(27,29)$. In addition, bintrafusp alfa showed a manageable safety profile and early signs of clinical efficacy in a phase I study of patients with heavily pretreated advanced solid tumors (NCT02517398; ref. 28). The objective of this trial was to assess the safety and tolerability of bintrafusp alfa in Asian patients with heavily pretreated advanced GC/GEJC.

\section{Patients and Methods \\ Study design and patients}

NCT02699515 is an ongoing, open-label, phase I trial of bintrafusp alfa with multiple solid tumor expansion cohorts. This report describes an expansion cohort of Asian patients with histologically or cytologically confirmed, recurrent or refractory, unresectable stage IV GC/GEJC for whom standard therapy does not exist or for whom standard therapy has failed. Tumor histology was initially captured using the Japanese or World Health Organization classification systems and later mapped to the Lauren classification system (Supplementary Table S1; refs. 30-33).

Eligible patients were ages $\geq 20$ years, had an Eastern Cooperative Oncology Group (ECOG) performance status $\leq 1$, and measurable disease by RECIST version 1.1 (RECIST 1.1). Patient selection was not based on PD-L1 expression or other biomarkers. Patients were ineligible for the study if they had active or previous central nervous system metastases or received prior treatment with ICIs or any therapy targeting TGF $\beta /$ TGF $\beta$ receptor. Full inclusion and exclusion criteria can be found in Supplementary Table S2.
This study was conducted in accordance with the ethical principles of the Declaration of Helsinki. Each patient provided written informed consent before study enrollment. Independent ethics committees or Institutional Review Boards at all participating institutions reviewed the study protocol with favorable opinions/approval. The study was conducted in accordance with international standards of good clinical practice consistent with the International Conference on Harmonisation Topic E6 Good Clinical Practice.

\section{Treatment and assessments}

Patients received bintrafusp alfa at the recommended phase II dose of $1,200 \mathrm{mg}$ via intravenous infusion over 1 hour once every 2 weeks until confirmed disease progression, unacceptable toxicity, or trial withdrawal (34). Tumor responses were assessed by CT or MRI every 6 weeks until disease progression during the treatment period and were evaluated according to RECIST 1.1. At the end of treatment, patients without progressive disease had assessments every 12 weeks. Dosing modifications, such as changes in infusion rate and dose delays, were permitted; however, dose reductions were not. All responses and disease progression were confirmed by additional scans, with an independent review committee (IRC) performing a blinded determination as to whether the criteria for response or progression had been met.

Adverse events (AE) were evaluated according to NCI-CTCAE v4.03. Immune-related AEs (irAE) were identified using a prespecified list of MedDRA terms and must have had onset after the first study drug administration and no more than 90 days after last dose. In addition, irAEs must have been treated with corticosteroids, immunosuppressants, or hormonal therapy, and have no clear etiology. Safety was assessed and documented throughout the trial, including monitoring for AEs, performance status evaluation, physical examination, and clinical laboratory tests. Electrocardiograms were performed at screening and at multiple treatment visits until week 13. After week 13, electrocardiograms were performed until disease progression, as well as at the end-oftreatment visit and safety follow-up visit. All patients had a visit scheduled for 10 weeks ( \pm 2 weeks) after the last administration of bintrafusp alfa for an assessment of safety parameters, including treatment-related AEs, concomitant medications, and further anticancer therapy.

\section{Endpoints}

The primary endpoint of this expansion cohort was safety/tolerability. Secondary endpoints included confirmed best overall response per RECIST 1.1, duration of response, disease control rate (DCR), PFS, and OS. Key exploratory endpoints were evaluation of potential predictive markers in tumors, including PD-L1 expression, EBV status, neoantigen count, MSI status, gene expression, and immune phenotype.

\section{Statistical analysis}

The goal of this expansion cohort was to explore the initial clinical activity of bintrafusp alfa in GC/GEJC and was viewed as hypothesisgenerating. Thirty patients were planned for this expansion cohort. The sample size was determined to obtain preliminary estimates of efficacy. The primary data cutoff date was 6 months after the last patient started treatment; the analysis presented here is a follow-up analysis. The ORR was determined as the proportion of patients with a confirmed best overall response of complete response (CR) or partial response (PR). The uncertainty of estimates was assessed by calculating a 95\% exact (Clopper-Pearson) CI. The DCR was defined as the 
proportion of patients with a confirmed best overall response of CR, $\mathrm{PR}$, stable disease (SD), or non-CR/non-progressive disease (PD). Duration of response, PFS, and OS were analyzed using the KaplanMeier method.

\section{Exploratory endpoints and procedures}

PD-L1 protein expression was detected using IHC staining of formalin-fixed, paraffin-embedded tumor tissue using an anti-PD-L1 rabbit $\mathrm{mAb}$ clone 22C3. PD-L1 expression was scored using the combined positive score (CPS), which represents the proportion of tumor and tumor-associated immune cells showing membranous PD-L1 staining. A threshold of 1 was used to classify samples as either PD-L1 positive $(\geq 1)$ or PD-L1 negative $(<1)$.

Gene expression analysis was performed using RNA sequencing (RNAseq) data that were generated from formalin-fixed, paraffinembedded archival tumor samples by Asuragen using standard protocols based on ribosomal depletion. Sequencing reads were aligned against the Ensembl 75 human genome (GRCh37 February 2014) using Bowtie2 version 2.2.3 (Johns Hopkins University, Baltimore, $\mathrm{MD}$; ref. 35). Gene expression was determined using RSEM version 1.2.31 with Ensembl gene annotations. Hypothesis testing was performed by comparing RSEM-computed expected counts (36). Transcript-per-million values were upper-quartile normalized and $\log$ transformed for further analysis.

Tumor EBV status was determined by RNAseq. Kallisto46 version 0.43.1 was used to align RNAseq reads to a transcriptome containing the human Ensembl transcripts (version 91, GRCh38) and viral genomes from the National Center for Biotechnology Information collection. The EBV content in each sample was assessed as the fraction of reads mapping to any EBV viral genome. To determine a cutoff (in terms of fraction of viral reads), the same pipeline was applied to publicly available samples from SRP055009 and SRP151215, which included EBV-transformed cell lines and were used as positive controls for viral transformation (37-39).

Tumor neoantigen count was measured using by RNAseq by performing a total RNA extraction, random priming library preparation, and ribosomal depletion by Asuragen. Tumor samples were sequenced at $2 \times 50$ to a target of $10^{8}$ read pairs on an Illumina HiSeq System (Illumina). Whole-exome sequencing was performed by Expression Analysis from matched peripheral blood samples using an Agilent SureSelect Human All Exon V5 Kit (Agilent Technologies); sequencing was done on an Illumina HiSeq System with a target of $100 \times$ coverage. RNAseq reads were mapped to hg19 and the Ensembl gene annotations (ensGene; University of California, Santa Cruz, CA) using RNA-STAR version 2.5.0b and whole-exome reads were mapped to hg19 using BWA-MEM version 0.7.12 $(40,41)$. Mutation calling was performed on paired BAM files (RNAseq tumor and whole-exome normal samples) using VarDictJava version 1.4.2, and the resulting mutations were annotated using Ensembl Variant Effect Predictor version 85 to determine the location and type of mutation $(42,43)$. HLA typing was performed on the RNAseq data using OptiType version 1.0.1. The HLA typing and mutation information were aggregated using a custom Python program which then ran NetMHCPan version 3.0 to determine the mutant and wild-type $\mathrm{IC}_{50} \mathrm{~s}$ of peptides, including the mutation site. Following filtering of neoantigens with an $\mathrm{IC}_{50}$ $>500 \mathrm{nmol} / \mathrm{L}$, a neoantigen count was produced for each sample. Furthermore, an expression-adjusted neoantigen score was produced that weighted each neoantigen according to its expression level. Finally, the per-sample values were normalized across the dataset by inverse of the number of reads mapped to genes in each sample.

MSI status was determined using the IdyllaTM MSI Assay (Biocartis) according to the manufacturer's specifications using patient tumor samples (44).

Tumor samples were used to determine immune phenotype based on available IHC data (PD-L1 stain and PD-L1 negative control) and hematoxylin and eosin staining. An exploratory classification system was used to categorize tumors as having an immune-inflamed (immune cells in direct physical contact with tumor cells), immune-excluded ( $\geq 1 \%$ of tumor stroma area populated by lymphocytes, immune cells possibly located in immediate vicinity of tumor cells but not efficiently infiltrating tumor cell clusters, and very infrequent physical contact between lymphocytes and tumor cells), or immune desert $(<1 \%$ of tumor stroma area populated by lymphocytes, no dense immune cell infiltrates, and no contact of immune cells with tumor cells) phenotype. This system was developed from methods previously described by Mariathasan and colleagues (22). A pathologist who was masked to the response data scored the scanned slides and determined the corresponding immune phenotype.

\section{Availability of materials and data}

For all new products or new indications approved in both the European Union and the United States after January 1, 2014, Merck KGaA, Darmstadt, Germany will share patient-level and study-level data after deidentification, as well as redacted study protocols and clinical study reports from clinical trials in patients. Any requests for these data by qualified scientific and medical researchers for legitimate research purposes will be subject to Merck KGaA's Data Sharing Policy. All requests should be submitted in writing to Merck KGaA's data sharing portal (https:// www.merckgroup.com/en/research/our-approach-to-research-anddevelopment/healthcare/clinical-trials/commitment-responsible-datasharing.html). When Merck KGaA has a coresearch, codevelopment, or comarketing or copromotion agreement, or when the product has been out-licensed, the responsibility for disclosure might be dependent on the agreement between parties. Under these circumstances, Merck $\mathrm{KGaA}$ will endeavor to gain agreement to share data in response to requests.

\section{Results}

\section{Patient demographics and treatment}

From January 11, 2017 to August 7, 2017, 44 patients were screened and 31 were enrolled at up to 30 trial centers in Japan, South Korea, and Taiwan. The median age was 64 years (range, 45-82) and most patients had an ECOG performance status of 1 $[n=23(74 \%)]$. All patients had previous anticancer therapy, with the majority receiving $\geq 3$ prior lines of treatment for locally advanced and/or metastatic disease $[n=19(61 \%)]$. In addition, $74 \%$ of patients $(n=23)$ had a PD-L1 CPS $\geq 1$ and were characterized as having PD-L1-positive tumors (Table 1). Twelve patients (39\%) had tumors with PD-L1 CPS $\geq 10$.

As of July 23, 2018, patients received bintrafusp alfa for a median of 10.1 weeks (range, 2.0-72.0) and the Kaplan-Meier estimate of median follow-up was 10.1 months (range, $0.5-16.8)$. Three patients (10\%) remained on treatment; disease progression $[n=20(65 \%)]$ was the most common primary reason for treatment termination (Supplementary Fig. S1). 
Table 1. Baseline patient and disease characteristics.

\begin{tabular}{|c|c|}
\hline Characteristic & $\boldsymbol{N}=\mathbf{3 1}$ \\
\hline \multicolumn{2}{|l|}{ Sex, $n(\%)$} \\
\hline Male & $26(84)$ \\
\hline Female & $5(16)$ \\
\hline Age, median (range), years & $64(45-82)$ \\
\hline \multicolumn{2}{|c|}{$\begin{array}{l}\text { Number of prior lines of therapy for advanced/metastatic } \\
\text { disease, } n(\%)^{\mathrm{a}}\end{array}$} \\
\hline $1^{\mathrm{b}}$ & $4(13)$ \\
\hline 2 & $8(26)$ \\
\hline$\geq 3$ & $19(61)$ \\
\hline \multicolumn{2}{|c|}{ ECOG performance status, $n(\%)$} \\
\hline 0 & $8(26)$ \\
\hline 1 & $23(74)$ \\
\hline \multicolumn{2}{|l|}{ Histology, $n(\%)^{c}$} \\
\hline Intestinal & $14(45)$ \\
\hline Diffuse & $2(6)$ \\
\hline Indeterminate & $7(23)$ \\
\hline Missing & $8(26)$ \\
\hline \multicolumn{2}{|l|}{ PD-L1 expression, $n$ (\%) } \\
\hline Positive & $23(74)$ \\
\hline Negative & $6(19)$ \\
\hline $\mathrm{NE}$ & $2(6)$ \\
\hline \multicolumn{2}{|l|}{ HER2 status, $n$ (\%) } \\
\hline 0 & $11(35)$ \\
\hline $1+$ & $6(19)$ \\
\hline $2+$ & $1(3)$ \\
\hline $3+$ & $3(10)$ \\
\hline NE & $10(32)$ \\
\hline \multicolumn{2}{|l|}{ EBV status, $n(\%)$} \\
\hline Positive & $3(10)$ \\
\hline Negative & $28(90)$ \\
\hline
\end{tabular}

Abbreviation: NE, not evaluable.

${ }^{a}$ Neoadjuvant and adjuvant therapy were included if disease progression occurred within 6 months of the treatment start date.

${ }^{b}$ One patient had incomplete dates recorded for adjuvant therapy and was considered as having only one prior line of therapy.

cHistology was initially captured using the Japanese or World Health Organization classification systems and later mapped to the Lauren classification system (Supplementary Table S1)

\section{Safety}

Among 31 patients, 16 (52\%) experienced a treatment-related $\mathrm{AE}$ (TRAE) of any grade, most commonly rash maculopapular $[n=7$ $(23 \%)]$, rash $[n=5(16 \%)]$, pruritus $[n=3(10 \%)]$, and fatigue $[n=3$ (10\%; (Table 2)]. Grade 3 TRAEs occurred in 6 patients (19\%), including anemia $[n=2(6 \%)]$, diarrhea, hepatic function abnormal, rash, rash maculopapular, and fracture $[n=1$ (3\%) each]. Two of these events (hepatic function abnormal and fracture) led to permanent treatment discontinuation. No patient experienced a grade 4 TRAE. On-treatment death occurred in 1 patient $(3 \%$; sudden death) after 5 doses of bintrafusp alfa. The cause of death was assessed by the investigator as treatment-related, confounded by suspected rupture of a preexisting thoracic aortic aneurysm that measured approximately $50 \mathrm{~mm}$ and was thought to be stable. Further radiographic diagnostic information was not provided, and no concomitant medication was recorded. No irAEs were reported by this patient. An autopsy was refused by the family, and therefore the definitive cause of death could not be determined. One patient (3\%) experienced a potentially TGF $\beta$-related skin lesion (keratoacanthoma of maximum grade 2), which was well managed with surgical excision and did not lead to treatment
Table 2. Incidence of TRAEs of any grade occurring in $\geq 5 \%$ of patients or of grade $\geq 3$.

\begin{tabular}{lllll}
\hline $\boldsymbol{n}(\%)$ & Any grade & Grade $\mathbf{3}$ & Grade $\mathbf{4}$ & Grade $\mathbf{5}$ \\
\hline Any TRAE & $16(52)$ & $6(19)$ & 0 & $1(3)$ \\
Rash maculopapular & $7(23)$ & $1(3)$ & 0 & 0 \\
Rash & $5(16)$ & $1(3)$ & 0 & 0 \\
Fatigue & $3(10)$ & 0 & 0 & 0 \\
Pruritus & $3(10)$ & 0 & 0 & 0 \\
Anemia & $2(6)$ & $2(6)$ & 0 & 0 \\
Diarrhea & $2(6)$ & $1(3)$ & 0 & 0 \\
Infusion-related reaction & $2(6)$ & 0 & 0 & 0 \\
Hepatic function abnormal & $1(3)$ & $1(3)$ & 0 & 0 \\
Fracture $^{\mathrm{a}}$ & $1(3)$ & $1(3)$ & 0 & 0 \\
Sudden death & $1(3)$ & 0 & 0 & $1(3)$ \\
Skin lesions $^{\mathrm{c}}$ & $1(3)$ & 0 & 0 & 0 \\
\hline
\end{tabular}

an a 70-year-old patient due to osteoporosis and long-term use of rosuvastatin calcium as a cosuspect drug, as conferred by the investigator.

${ }^{b}$ The cause of death, assessed by the investigator as treatment related, was suspected to be rupture of a preexisting thoracic aortic aneurysm

'Includes actinic keratosis, basal cell carcinoma, hyperkeratosis, keratoacanthoma, and squamous cell carcinoma of the skin NCI-CTCAE v4.03 preferred terms. One patient (3\%) had grade 2 keratoacanthoma.

discontinuation. Infusion-related reactions occurred in 2 patients (6\%; grades 1 and 2), which resolved and did not result in treatment discontinuation. Ten patients $(32 \%)$ had at least one irAE; immune-related rash occurred in all patients with an irAE. No irAEs were above grade 3 .

\section{Efficacy}

As per IRC assessment, responses were confirmed in 5 of 31 patients [16\% (95\% CI, 6-34)], including one CR. The DCR was $26 \%$ (95\% CI, $12-$ 45; Table 3; Fig. 1). There was good concordance between IRC- and investigator-assessed best overall response (Supplementary Table S3). Median duration of response was 8.7 months (range, 2.4-12.4+), with one PR ongoing for $12.4+$ months at the time of data cutoff. An additional patient with a PR had a duration of response of 6.9 months that was considered ongoing as of the last assessment; however, the patient was later lost to follow-up and discontinued treatment due to AEs.

The median PFS per IRC assessment was 1.3 months (95\% CI, 1.2$2.6)$, with a 6-month rate of $17 \%(95 \% \mathrm{CI}, 6-32)$ and 12 -month rate of $11 \%$ (95\% CI, 3-27). Median PFS was 2.1 months (95\% CI, 1.2-3.9) per investigator assessment (Supplementary Fig. S2). The median OS was 10.1 months (95\% CI, 4.5-12.8), with 6- and 12-month rates of $63 \%$ (95\% CI, 43-77) and 41\% (95\% CI, 23-58), respectively (Fig. 2).

Exploratory analysis showed PD-L1 expression was not predictive of response (Table 3; Fig. 3) and did not impact survival (Fig. 2). The IRC-assessed ORR was similar for patients with PD-L1-positive disease, regardless of CPS cutoff [CPS $\geq 1,17 \%$ ( $n=4$ of 23 ); CPS $\geq 10,17 \%$ ( $n=2$ of 12$)]$. There was also no strong association between response and HER2 status, EBV infection, tumor neoantigen count, or MSI-high status (Table 3; Fig. 3). Interestingly, high expression of TGFB1 in tumor samples enriched for response to bintrafusp alfa (Fig. 3). In addition, responses were most common $(n=4)$ in tumors with an inflamed immune phenotype (Supplementary Fig. S3).

\section{Discussion}

In this first evaluation of bintrafusp alfa in Asian patients with heavily pretreated advanced GC/GEJC ( $61 \%$ received $\geq 3$ prior lines of 
Kang et al.

Table 3. Efficacy outcomes.

\begin{tabular}{|c|c|c|}
\hline & $\begin{array}{l}\text { IRC } \\
(N=31)\end{array}$ & $\begin{array}{l}\text { Investigator } \\
(N=31)\end{array}$ \\
\hline \multicolumn{3}{|c|}{ Confirmed best overall response, $n(\%)$} \\
\hline $\mathrm{CR}$ & $1(3)$ & $2(6)$ \\
\hline PR & $4(13)$ & $5(16)$ \\
\hline $\mathrm{SD}$ & $2(6)$ & $5(16)$ \\
\hline Non-CR/non-PD & $1(3)$ & 0 \\
\hline PD & $21(68)$ & $16(52)$ \\
\hline NE & $2(6)$ & $3(10)$ \\
\hline ORR, $n(\%, 95 \% \mathrm{Cl})$ & $5(16,6-34)$ & $7(23,10-41)$ \\
\hline DCR, $n(\%, 95 \% \mathrm{Cl})$ & $8(26,12-45)$ & $12(39,22-58)$ \\
\hline Median duration of response, mo & 8.7 & 9.0 \\
\hline Range & $2.4-12.4+$ & $2.4-12.3+$ \\
\hline \multicolumn{3}{|l|}{ ORR by PD-L1 expression, $n(\%)$} \\
\hline Positive $(n=23)$ & $4(17)$ & $4(17)$ \\
\hline Negative $(n=6)$ & $1(17)$ & $2(33)$ \\
\hline $\mathrm{NE}(n=2)$ & 0 & $1(50)$ \\
\hline \multicolumn{3}{|l|}{ ORR by HER2 status, $n$ (\%) } \\
\hline $0(n=11)$ & $2(18)$ & $2(18)$ \\
\hline $1+(n=6)$ & $1(17)$ & $1(17)$ \\
\hline $2+(n=1)$ & 0 & 0 \\
\hline $3+(n=3)$ & $1(33)$ & $2(67)$ \\
\hline $\mathrm{NE}(n=10)$ & $1(10)$ & $2(20)$ \\
\hline \multicolumn{3}{|l|}{ ORR by MSI status, $n$ (\%) } \\
\hline $\mathrm{MSI}-\mathrm{H}(n=1)$ & 0 & 0 \\
\hline $\operatorname{MSS}(n=13)$ & $2(15)$ & $3(23)$ \\
\hline $\mathrm{NE}(n=17)$ & $3(18)$ & $4(24)$ \\
\hline
\end{tabular}

Abbreviations: MSI-H, microsatellite instability-high; MSS, microsatellite stable; NE, not evaluable.

${ }^{a}$ Non-CR/non-PD applies to patients with no target lesions and is defined as persistence of $\geq 1$ nontarget lesion(s).

therapy for locally advanced and/or metastatic disease), treatment had a manageable safety profile with $19 \%$ of patients experiencing a grade 3 TRAE and no grade 4 events. One treatment-related death occurred in a patient with a history of thoracic aortic aneurysms. Rashes, including rash maculopapular, were the most common TRAEs; however, these events were mild and resolved with simple medical measures. Rashes were not assessed as infusion-related reactions but were a common irAE. Skin lesions, which are anticipated AEs with TGF $\beta$ inhibition and other kinase inhibitors which may impact TGF $\beta$ signaling, were reported in 1 patient and did not lead to treatment discontinuation (45). Overall, the safety profile in this cohort was consistent with previously reported data for bintrafusp alfa in other solid tumors (28). In addition, the safety profile of bintrafusp alfa was comparable with that reported in phase II and III trials of PD-1 inhibitors in similar populations (18-20).

Treatment with bintrafusp alfa resulted in a median OS of 10.1 months and ORR of $16 \%$. These outcomes compare favorably with historical findings from large phase II and III studies of internationally recommended treatments for pretreated GC/GEJC, such as TAS-102 and PD-1 inhibitors, in the overall PD-L1unselected population. In these studies, including the phase III ATTRACTION-2 study which evaluated Asian patients, median OS ranged from 5.3 to 6.7 months and ORRs were between $4 \%$ and $12 \%(17-20)$. Although results with bintrafusp alfa are encouraging, it is important to note that results of this single-arm, phase I study cannot be compared directly with these historical findings due to key differences in study design, eligibility criteria, and patient populations. Larger studies with an active comparator are required to determine the clinical benefit of dual inhibition of TGF $\beta$ and PDL1 in GC/GEJC.

Unlike results for PD-1 inhibitors in GC/GEJC, there was no clear association between efficacy of bintrafusp alfa and PD-L1 expression $(19,20,46)$. While the results must be interpreted cautiously due to the small number of patients, the IRC-assessed ORR was $17 \%$ for PD-L1-negative patients. Future studies are needed to confirm whether bintrafusp alfa could provide therapeutic benefit for patients who do not typically respond to PD-1 inhibitors, such as PD-L1-negative patients. In addition, EBV-positivity and MSI status did not predict response to bintrafusp alfa $(6,19,20,46)$.

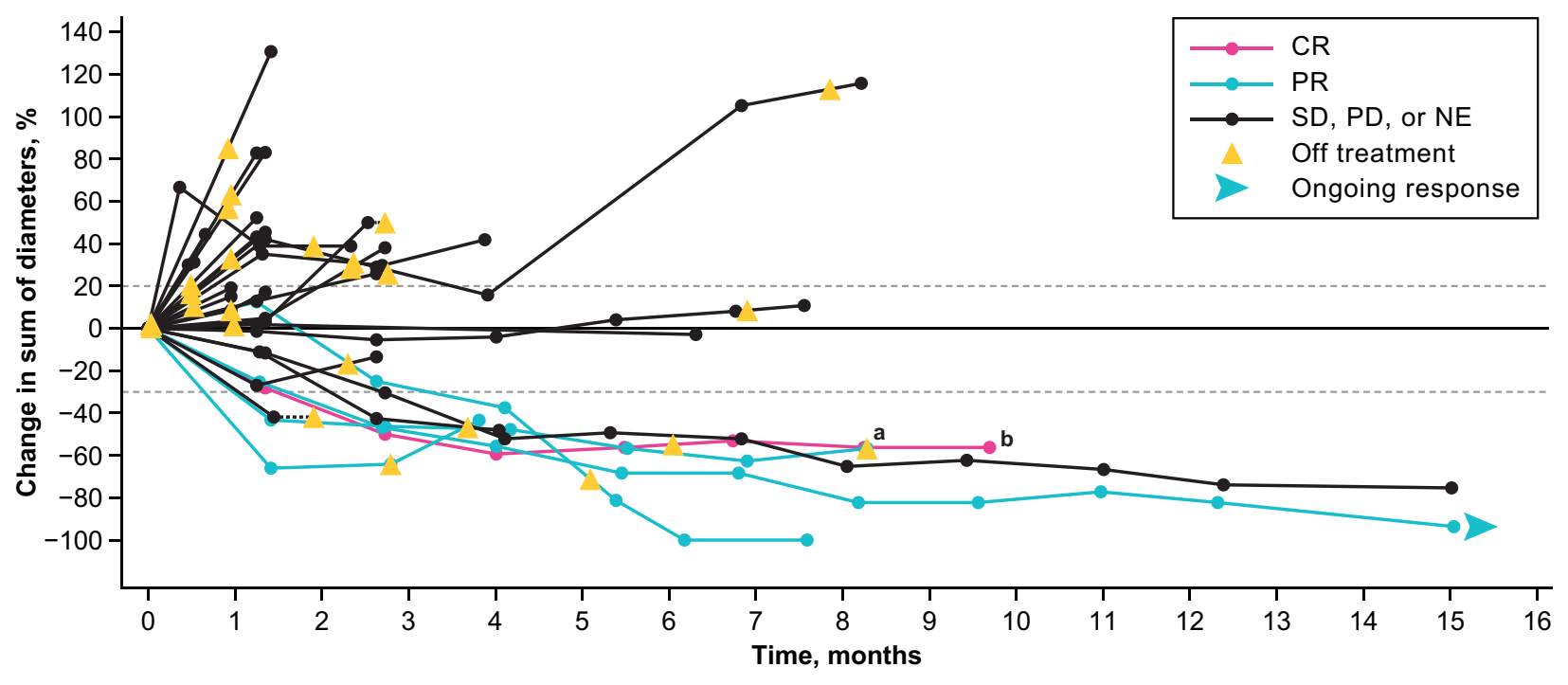

Figure 1.

Change in target lesions by IRC assessment. Two patients are not shown due to either no postbaseline assessment or no target lesions identified prior to the first dose. $\mathrm{NE}$, not evaluable. ${ }^{\mathrm{a}}$ Patient was lost to follow-up after the last assessment of PR and later discontinued treatment. ${ }^{\mathrm{b}}$ Percent change in sum of diameters was not $-100 \%$ because patient had only lymph node lesions. 
Bifunctional Targeting of TGF $\beta$ and PD-L1 in GC with Bintrafusp Alfa

A

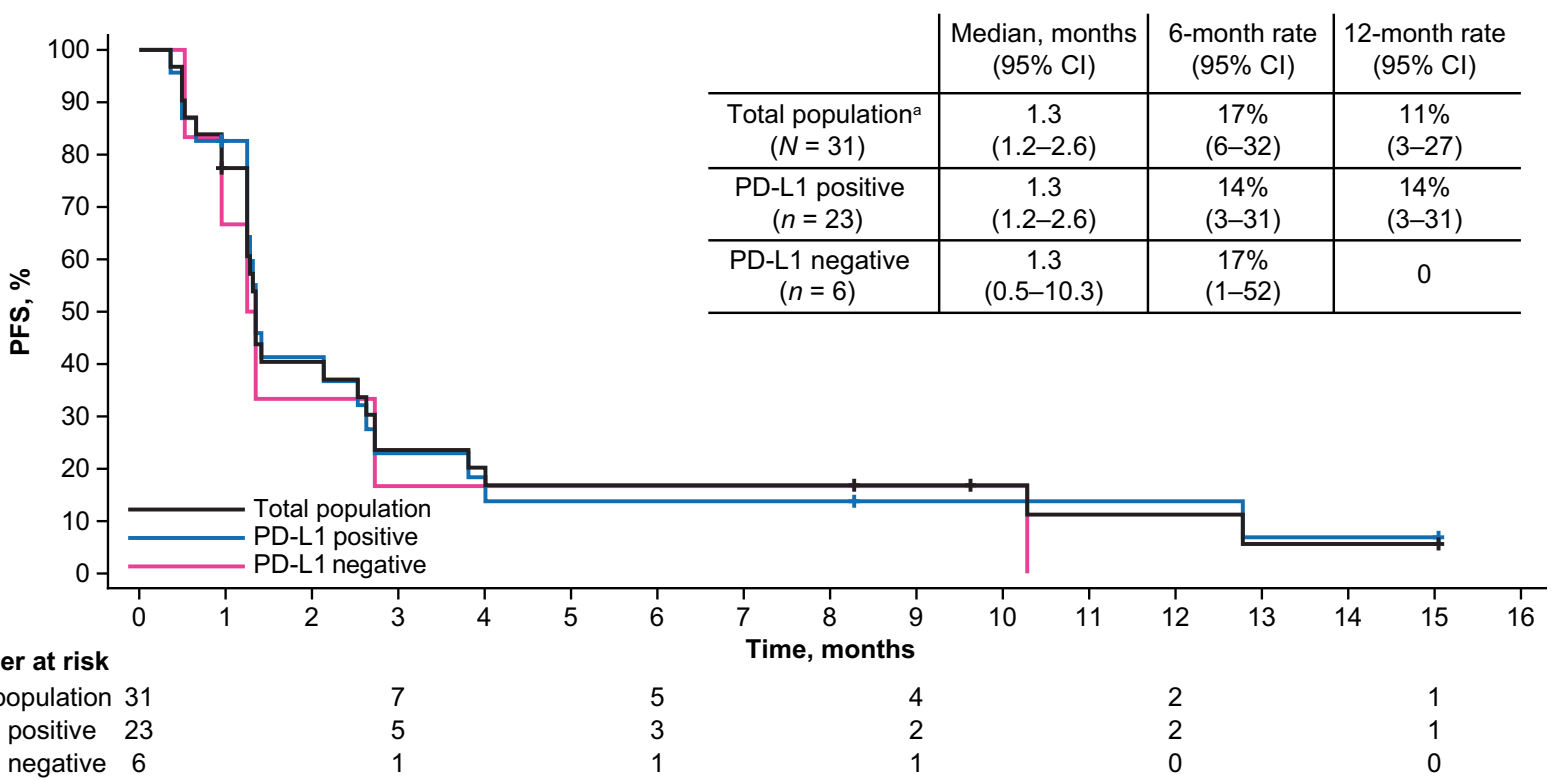

B

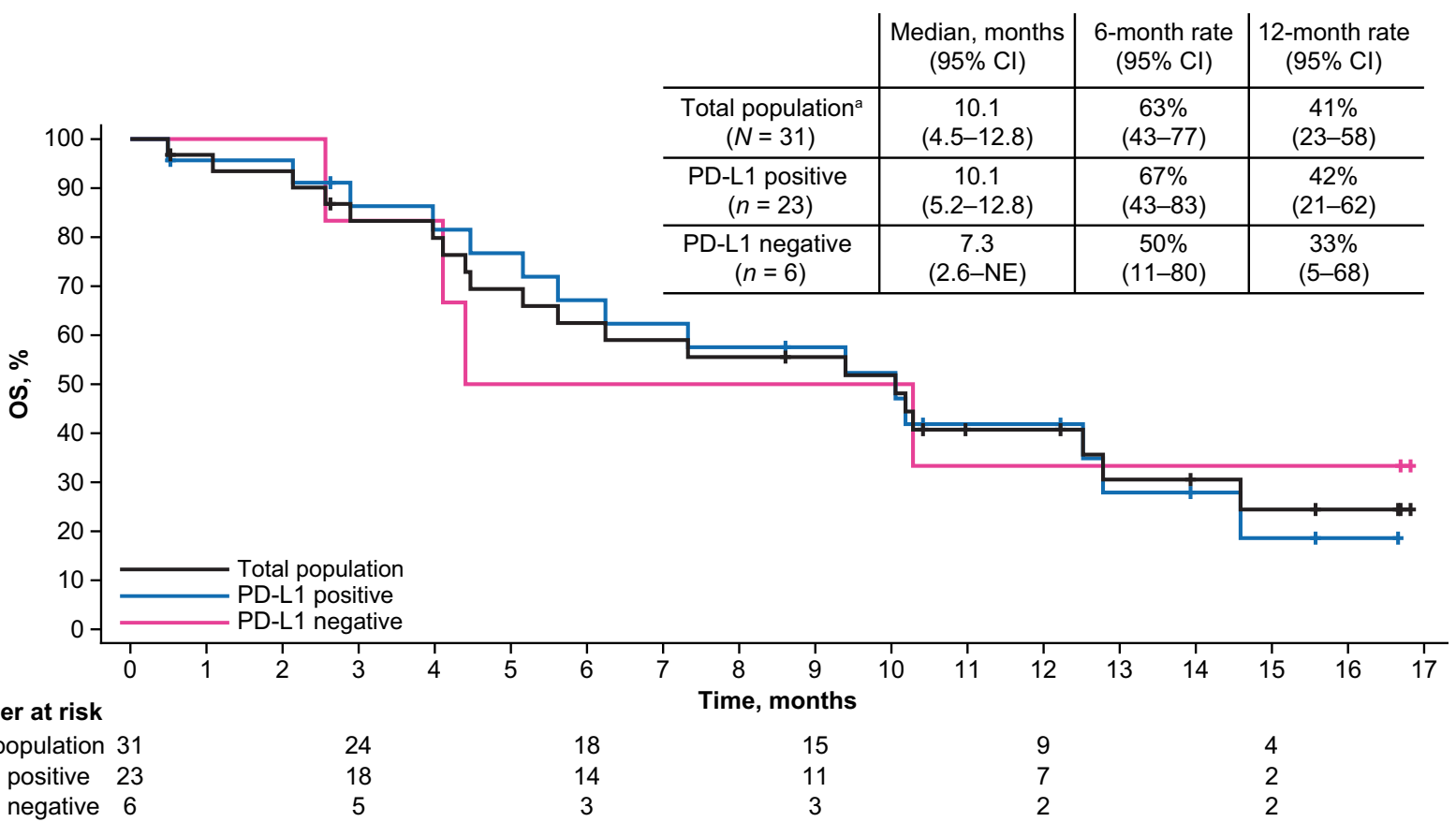

Number at risk

Total population 31

PD-L1 positive 23

5

18
14
3

3

2

Figure 2.

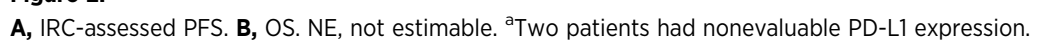

Results of exploratory analysis revealed responses to bintrafusp alfa were enriched in patients with high tumor TGFB1 levels, possibly underscoring the value of dual inhibition of both the TGF $\beta$ and PD-L1 pathways as a potential therapeutic option for patients with GC/GEJC. Larger studies will be needed to confirm this observation and to determine whether TGFB1 expression correlated with other pathologic features or efficacy outcomes. Furthermore, additional analyses, including a comparison of tumor TGFB1 expression before, during, and after treatment, are of interest.

In conclusion, bintrafusp alfa had a manageable safety profile and showed signs of clinical activity by eliciting durable responses in this phase I study of Asian patients with heavily pretreated advanced GC/GEJC. Further study of bintrafusp alfa in patients with advanced $\mathrm{GC} / \mathrm{GEJC}$ is warranted to confirm these findings. 
Kang et al.
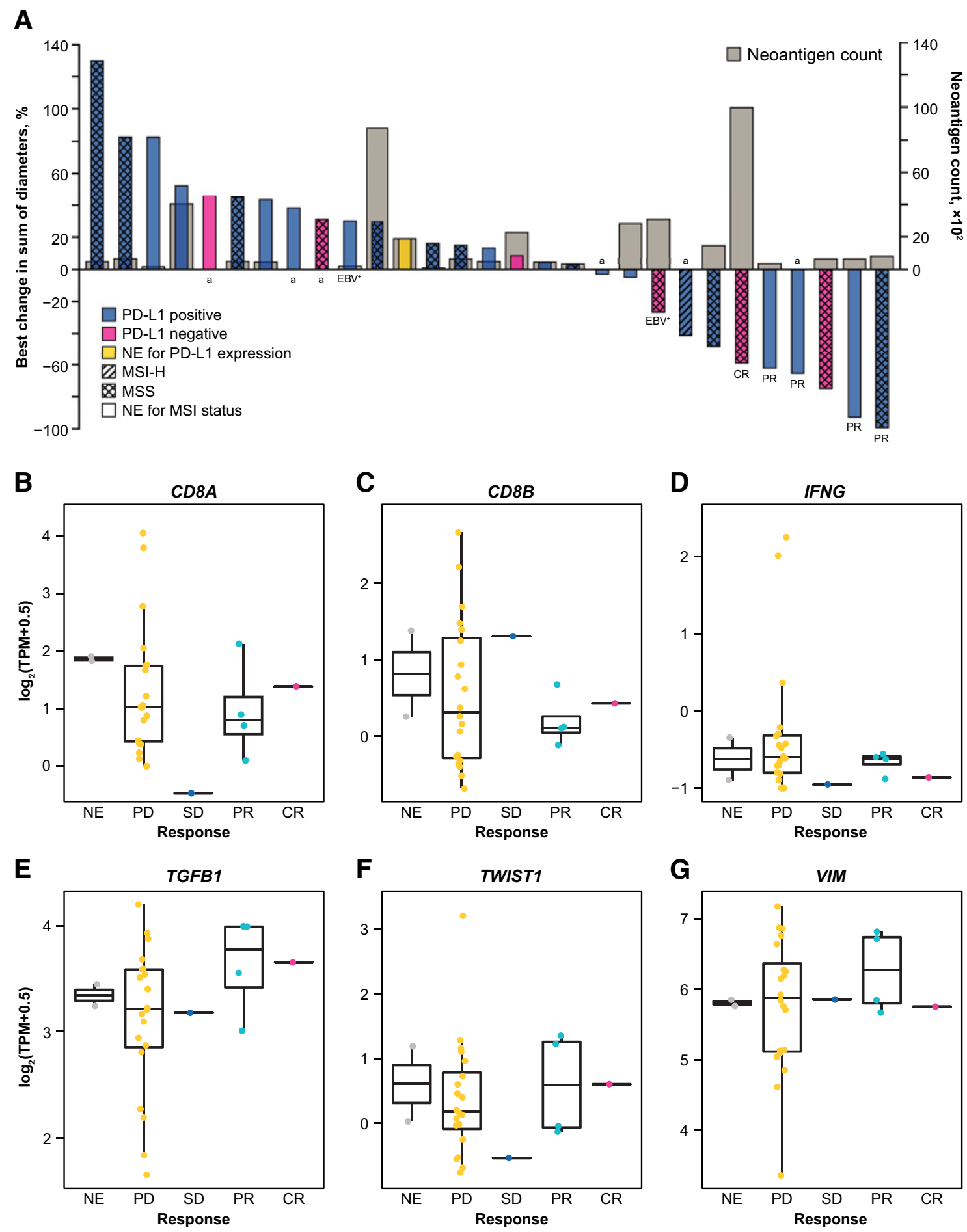

Figure 3.

A, Best change in sum of diameters per IRC assessment and neoantigen count. Two patients, including 1 with EBV-positive disease, are not shown due to either no postbaseline assessment or no target lesions identified prior to the first dose. B-G, RNAseq analysis by IRC-assessed best overall response. One patient with a confirmed best overall response of PD whose tumor sample had abnormally few detectable genes in RNAseq, and 1 patient with a confirmed best overall response of SD for which no RNAseq data were obtained, were excluded from further biomarker analyses. CD8A, CD8a molecule; CD8B, CD8b molecule; IFNG, interferon gamma; MSI-H, microsatellite instability-high; MSS, microsatellite stable; NE, not evaluable; TGFB1, transforming growth factor beta 1; TPM, transcript-per-million; TWIST1, twist family bHLH transcription factor 1; VIM, vimentin. ${ }^{a}$ Tumor neoantigen count unavailable. 


\section{Disclosure of Potential Conflicts of Interest}

Y-J. Bang is an employee/paid consultant for MSD, AstraZeneca, Genentech, Merck Serono, BeiGene, ALX Oncology Inc. and Bristol Myers Squibb. K. Muro reports receiving speakers bureau honoraria from Taiho Pharmaceutical Co., LTD., Chuga Pharmaceutical Co., LTD., Takeda Pharmaceutical Co., LTD., Ono Pharmaceutical Co., LTD., MSD, Bristol Myers Squibb, Sanofi, Bayer, and Eli Lilly. I. Dussault is an employee/paid consultant for EMD Serono and holds ownership interest (including patents) in EMD Serono, Bristol Myers Squibb, and Amgen. C. Helwig is an employee/ paid consultant for and holds ownership interest (including patents) in Merck KGaA Darmstadt, Germany. M. Osada is an employee/paid consultant for Merck Biopharma. No potential conflicts of interest were disclosed by the other authors.

\section{Authors' Contributions}

Conception and design: Y.-J. Bang, I. Dussault, C. Helwig, M. Osada, T. Doi Development of methodology: Y.-J. Bang, I. Dussault, C. Helwig, M. Osada Acquisition of data (provided animals, acquired and managed patients, provided facilities, etc.): Y.-K. Kang, Y.-J. Bang, S. Kondo, H.C. Chung, K. Muro, I. Dussault, T. Doi

Analysis and interpretation of data (e.g., statistical analysis, biostatistics, computational analysis): Y.-K. Kang, Y.-J. Bang, H.C. Chung, I. Dussault, C. Helwig, M. Osada, T. Doi

Writing, review, and/or revision of the manuscript: Y.-K. Kang, Y.-J. Bang,

S. Kondo, H.C. Chung, K. Muro, I. Dussault, C. Helwig, M. Osada, T. Doi

\section{References}

1. International Agency for Research on Cancer. Stomach; 2019. Available from: http://gco.iarc.fr/today/data/factsheets/cancers/7-Stomach-fact-sheet.pdf.

2. Muro K, Van Cutsem E, Narita Y, Pentheroudakis G, Baba E, Li J, et al. Pan-Asian adapted ESMO Clinical Practice Guidelines for the management of patients with metastatic gastric cancer: a JSMO-ESMO initiative endorsed by CSCO, KSMO, MOS, SSO and TOS. Ann Oncol 2019;30:19-33.

3. Wagner AD, Syn NL, Moehler M, Grothe W, Yong WP, Tai BC, et al. Chemotherapy for advanced gastric cancer. Cochrane Database Syst Rev 2017;8:CD004064.

4. Shitara K, Matsuo K, Muro K, Doi T, Ohtsu A. Progression-free survival and post-progression survival in patients with advanced gastric cancer treated with first-line chemotherapy. J Cancer Res Clin Oncol 2013;139:1383-9.

5. NCCN Clinical Practice Guidelines in Oncology. Gastric Cancer; 2019. Available from: https://www.nccn.org/professionals/physician_gls/pdf/gastric.pdf.

6. Kim ST, Cristescu R, Bass AJ, Kim KM, Odegaard JI, Kim K, et al. Comprehensive molecular characterization of clinical responses to PD-1 inhibition in metastatic gastric cancer. Nat Med 2018;24:1449-58.

7. Chen DS, Mellman I. Elements of cancer immunity and the cancer-immune set point. Nature 2017;541:321-30.

8. Boger C, Behrens HM, Mathiak M, Kruger S, Kalthoff H, Rocken C. PD-L1 is an independent prognostic predictor in gastric cancer of Western patients. Oncotarget 2016;7:24269-83.

9. Lee HE, Chae SW, Lee YJ, Kim MA, Lee HS, Lee BL, et al. Prognostic implications of type and density of tumour-infiltrating lymphocytes in gastric cancer. $\mathrm{Br} \mathrm{J}$ Cancer 2008;99:1704-11.

10. Joyce JA. Therapeutic targeting of the tumor microenvironment. Cancer Cell 2005;7:513-20.

11. Principe DR, Doll JA, Bauer J, Jung B, Munshi HG, Bartholin L, et al. TGF- $\beta$ : duality of function between tumor prevention and carcinogenesis. J Natl Cancer Inst 2014;106:djt369.

12. Akhurst RJ, Hata A. Targeting the TGF $\beta$ signalling pathway in disease. Nat Rev Drug Discov 2012;11:790-811.

13. Colak S, ten Dijke P. Targeting TGF- $\beta$ signaling in cancer. Trends Cancer 2017;3: $56-71$.

14. Saito H, Tsujitani S, Oka S, Kondo A, Ikeguchi M, Maeta M, et al. The expression of transforming growth factor- $\beta 1$ is significantly correlated with the expression of vascular endothelial growth factor and poor prognosis of patients with advanced gastric carcinoma. Cancer 1999;86:1455-62.

15. Nakamura M, Katano M, Kuwahara A, Fujimoto K, Miyazaki K, Morisaki T, et al Transforming growth factor $\beta 1$ (TGF- $\beta 1$ ) is a preoperative prognostic indicator in advanced gastric carcinoma. Br J Cancer 1998;78:1373-8.
Administrative, technical, or material support (i.e., reporting or organizing data, constructing databases): M. Osada

Study supervision: Y.-J. Bang

\section{Acknowledgments}

The authors thank the patients, investigators, coinvestigators, and the study teams at each of the participating centers and at Merck KGaA, Darmstadt, Germany. The authors thank Christian Ihling of Merck KGaA for his substantial contribution to the immune phenotype analysis. This trial was funded by Merck KGaA and is part of an alliance between Merck KGaA and GlaxoSmithKline. Medical writing support was provided by Lauren Rusnak, $\mathrm{PhD}$, of ClinicalThinking, Inc, and funded by Merck KGaA and GlaxoSmithKline in accordance with Good Publication Practice (GPP3) guidelines (http://www.ismpp.org/gpp3).

The costs of publication of this article were defrayed in part by the payment of page charges. This article must therefore be hereby marked advertisement in accordance with 18 U.S.C. Section 1734 solely to indicate this fact.

Received November 22, 2019; revised March 3, 2020; accepted April 10, 2020; published first April 16, 2020.
16. Peng LS, Zhang JY, Teng YS, Zhao YL, Wang TT, Mao FY, et al. Tumorassociated monocytes/macrophages impair NK-cell function via TGF $\beta 1$ in human gastric cancer. Cancer Immunol Res 2017;5:248-56.

17. Shitara K, Doi T, Dvorkin M, Mansoor W, Arkenau HT, Prokharau A, et al Trifluridine/tipiracil versus placebo in patients with heavily pretreated metastatic gastric cancer (TAGS): a randomised, double-blind, placebo-controlled phase 3 trial. Lancet Oncol 2018;19:1437-48.

18. Kang YK, Boku N, Satoh T, Ryu MH, Chao Y, Kato K, et al. Nivolumab in patients with advanced gastric or gastro-oesophageal junction cancer refractory to, or intolerant of, at least two previous chemotherapy regimens (ONO-4538-12, ATTRACTION-2): a randomised, double-blind, placebo-controlled, phase 3 trial. Lancet 2017;390:2461-71.

19. Fuchs CS, Doi T, Jang RW, Muro K, Satoh T, Machado M, et al. Safety and efficacy of pembrolizumab monotherapy in patients with previously treated advanced gastric and gastroesophageal junction cancer: phase 2 clinical KEYNOTE-059 trial. JAMA Oncol 2018;4:e180013.

20. Shitara K, Özgüroğlu M, Bang YJ, Di Bartolomeo M, Mandalà M, Ryu $\mathrm{MH}$, et al. Pembrolizumab versus paclitaxel for previously treated, advanced gastric or gastro-oesophageal junction cancer (KEYNOTE061): a randomised, open-label, controlled, phase 3 trial. Lancet 2018; 392:123-33.

21. Bang YJ, Ruiz EY, Van Cutsem E, Lee KW, Wyrwicz L, Schenker M, et al. Phase III, randomised trial of avelumab versus physician's choice of chemotherapy as third-line treatment of patients with advanced gastric or gastro-oesophageal junction cancer: primary analysis of JAVELIN Gastric 300. Ann Oncol 2018;29: 2052-60.

22. Mariathasan S, Turley SJ, Nickles D, Castiglioni A, Yuen K, Wang Y, et al. TGF $\beta$ attenuates tumour response to PD-L1 blockade by contributing to exclusion of T cells. Nature 2018;554:544-8.

23. Hanks BA, Holtzhausen A, Evans K, Heid M, Blobe GC. Combinatorial TGF- $\beta$ signaling blockade and anti-CTLA-4 antibody immunotherapy in a murine $\mathrm{BRAF}^{\mathrm{V600E}}-\mathrm{PTEN}^{-1-}$ transgenic model of melanoma. J Clin Oncol 32:15s, 2014 (suppl; abstr 3011)

24. Holmgaard RB, Schaer DA, Li Y, Castaneda SP, Murphy MY, Xu X, et al. Targeting the TGF $\beta$ pathway with galunisertib, a TGF $\beta$ RI small molecule inhibitor, promotes anti-tumor immunity leading to durable, complete responses, as monotherapy and in combination with checkpoint blockade. J Immunother Cancer 2018;6:47.

25. Tauriello DVF, Palomo-Ponce S, Stork D, Berenguer-Llergo A, Badia-Ramentol $\mathrm{J}$, Iglesias $\mathrm{M}$, et al. TGF $\beta$ drives immune evasion in genetically reconstituted colon cancer metastasis. Nature 2018;554:538-43. 
Kang et al.

26. Principe DR, Park A, Dorman MJ, Kumar S, Viswakarma N, Rubin J, et al. TGF $\beta$ blockade augments PD-1 inhibition to promote T-cell-mediated regression of pancreatic cancer. Mol Cancer Ther 2019;18:613-20.

27. Lan Y, Zhang D, Xu C, Hance KW, Marelli B, Qi J, et al. Enhanced preclinical antitumor activity of M7824, a bifunctional fusion protein simultaneously targeting PD-L1 and TGF- $\beta$. Sci Transl Med 2018;10:eaan5488.

28. Strauss J, Heery CR, Schlom J, Madan RA, Cao L, Kang Z, et al. Phase I trial of M7824 (MSB0011359C), a bifunctional fusion protein targeting PD-L1 and TGF $\beta$, in advanced solid tumors. Clin Cancer Res 2018;24:1287-95.

29. Knudson KM, Hicks KC, Luo X, Chen JQ, Schlom J, Gameiro SR. M7824, a novel bifunctional anti-PD-L1/TGF $\beta$ Trap fusion protein, promotes anti-tumor efficacy as monotherapy and in combination with vaccine. Oncoimmunology 2018 ; 7:e1426519.

30. Japanese Gastric Cancer Association. Japanese classification of gastric carcinoma: 3rd English edition. Gastric Cancer 2011;14:101-12.

31. Bosman FT, Carneiro F, Hruban RH, Theise ND, editors. WHO classification of tumors of the digestive system. 4th edition. Lyon, France: IARC Press; 2010.

32. Lauren P. The two histological main types of gastric carcinoma: diffuse and socalled intestinal-type carcinoma. an attempt at a histo-clinical classification. Acta Pathol Microbiol Scand 1965;64:31-49.

33. Berlth F, Bollschweiler E, Drebber U, Hoelscher AH, Moenig S. Pathohistological classification systems in gastric cancer: diagnostic relevance and prognostic value. World J Gastroenterol 2014;20:5679-84.

34. Vugmeyster Y, Wilkins J, Harrison-Moench E, Geng W, Koenig A, Cao L, et al. Selection of the recommended phase 2 dose (RP2D) for M7824 (MSB0011359C), a bifunctional fusion protein targeting TGF- $\beta$ and PD-L1. J Clin Oncol 36:18s, 2018 (suppl; abstr 2566).

35. Langmead B, Salzberg SL. Fast gapped-read alignment with Bowtie 2. Nat Methods 2012;9:357-9.

36. Li B, Dewey CN. RSEM: accurate transcript quantification from RNA-Seq data with or without a reference genome. BMC Bioinformatics 2011;12:323.
37. Kostic AD, Ojesina AI, Pedamallu CS, Jung J, Verhaak RGW, Getz G, et al PathSeq: software to identify or discover microbes by deep sequencing of human tissue. Nat Biotechnol 2011;29:393-6.

38. Salyakina D, Tsinoremas NF. Viral expression associated with gastrointestinal adenocarcinomas in TCGA high-throughput sequencing data. Hum Genomics 2013;7:23.

39. Tang KW, Alaei-Mahabadi B, Samuelsson T, Lindh M, Larsson E. The landscape of viral expression and host gene fusion and adaptation in human cancer. Nat Commun 2013;4:2513.

40. Dobin A, Davis CA, Schlesinger F, Drenkow J, Zaleski C, Jha S, et al. STAR: ultrafast universal RNA-seq aligner. Bioinformatics 2013;29:15-21.

41. Li H, Durbin R. Fast and accurate short read alignment with Burrows-Wheeler transform. Bioinformatics 2009;25:1754-60.

42. Lai Z, Markovets A, Ahdesmaki M, Chapman B, Hofmann O, McEwen R, et al. VarDict: a novel and versatile variant caller for next-generation sequencing in cancer research. Nucleic Acids Res 2016;44:e108.

43. McLaren W, Gil L, Hunt SE, Riat HS, Ritchie GRS, Thormann A, et al. The ensembl variant effect predictor. Genome Biol 2016;17:122.

44. De Craene B, Feng Z, Rondelez E, Vandenbroeck L, Peeters K, Maertens G, et al. Detection of microsatellite instability (MSI) with a novel panel of biomarkers in gastric cancer samples. Ann Oncol 28:5s, 2017 (suppl; abstr $\operatorname{mdx} 369.081$ ).

45. Lacouture ME, Morris JC, Lawrence DP, Tan AR, Olencki TE, Shapiro GI, et al. Cutaneous keratoacanthomas/squamous cell carcinomas associated with neutralization of transforming growth factor $\beta$ by the monoclonal antibody fresolimumab (GC1008). Cancer Immunol Immunother 2015;64: 437-46.

46. Kim JH, Ryu M-H, Park YS, Ma J, Lee SY, Kim D, et al. Predictive biomarkers for the efficacy of nivolumab as $\geq$ third-line therapy in patients with advanced gastric cancer (AGC): from a subset analysis of ATTRACTION-2 phase III trial. J Clin Oncol 37:4s, 2019 (suppl; abstr 152). 


\section{Clinical Cancer Research}

\section{Safety and Tolerability of Bintrafusp Alfa, a Bifunctional Fusion Protein Targeting TGF $\beta$ and PD-L1, in Asian Patients with Pretreated Recurrent or Refractory Gastric Cancer}

Yoon-Koo Kang, Yung-Jue Bang, Shunsuke Kondo, et al.

Clin Cancer Res 2020;26:3202-3210. Published OnlineFirst April 16, 2020.

\section{Updated version Access the most recent version of this article at: doi:10.1158/1078-0432.CCR-19-3806}

Supplementary Access the most recent supplemental material at:

Material http://clincancerres.aacrjournals.org/content/suppl/2020/04/16/1078-0432.CCR-19-3806.DC1

Cited articles This article cites 42 articles, 5 of which you can access for free at: http://clincancerres.aacrjournals.org/content/26/13/3202.full\#ref-list-1

E-mail alerts Sign up to receive free email-alerts related to this article or journal.

Reprints and To order reprints of this article or to subscribe to the journal, contact the AACR Publications Department at Subscriptions pubs@aacr.org.

Permissions To request permission to re-use all or part of this article, use this link http://clincancerres.aacrjournals.org/content/26/13/3202.

Click on "Request Permissions" which will take you to the Copyright Clearance Center's (CCC) Rightslink site. 